Z Gerontol Geriat 2014 · 47:192-193

DOI 10.1007/s00391-014-0649-2

Online publiziert: 5. April 2014

(c) Springer-Verlag Berlin Heidelberg 2014

Daniel Kopf ${ }^{1} \cdot$ Walter Hewer ${ }^{2}$

${ }^{1}$ Zentrum für klinische Psychologie und Psychotherapie, Marienkrankenhaus, Hamburg

${ }^{2}$ Abteilung Gerontopsychiatrie, Vinzenz von Paul Hospital gGmbH , Rottweil

\title{
Psychotraumatologie im Alter
}

Während des zweiten Weltkriegs und der frühen Nachkriegszeit waren unzählige Menschen, besonders in der Zivilbevölkerung, einer Vielzahl von traumatisierenden Ereignissen ausgesetzt. Für Menschen, die diese Zeit als Kinder, Jugendliche und junge Erwachsene erlebt haben, traten diese traumatischen Ereignisse in entscheidenden Phasen der psychischen Entwicklung auf. In Deutschland summierte sich die Zahl der Betroffenen von Luftangriffen, Flucht und Vertreibung, Verlust von Eltern im Krieg und sogar der Rekrutierung von Jugendlichen zum Wehrdienst zu einem hohen Anteil an der Gesamtbevölkerung. Bei der Volkszählung in der Bundesrepublik Deutschland 1950 waren von knapp 19 Mio. Menschen unter 25 Jahren etwa 3,3 Mio. Vertriebene und etwa 1,25 Mio. Menschen hatten ihren Vater durch den Krieg verloren. Davon waren 250.000 Vollwaisen $[1,6]$. Unzählige weitere hatten die Schrecken der Luftangriffe auf deutsche Großstädte oder Artilleriebeschuss erlebt. Die meisten Angehörigen dieser Generation haben bislang kaum erforschte Bewältigungsstrategien gefunden, die ihnen ein Leben mit zwischenmenschlichen Beziehungen in guter sozialer und beruflicher Integration und ohne relevante psychiatrische Morbidität ermöglicht haben. Heute sind diese Menschen in einem Alter, in dem sie durch körperliche Erkrankungen, altersbedingte Verluste an Funktionalität, Rollenwechsel und alterstypische Erkrankungen des Gehirns vor neue Herausforderungen gestellt werden. In geriatrischen und gerontopsychiatrischen Kliniken und Einrichtungen der Altenhilfe fällt in den letzten Jahren immer häu- figer auf, dass diese lange verschwiegenen traumatischen Erfahrungen aus der Kindheit und Jugend in belastender Weise zum Vorschein kommen: Sie werden von $\mathrm{Pa}-$ tienten entweder direkt thematisiert oder sie sind Gegenstand von Halluzinationen oder wahnhaften Verkennungen bei demenziellen und deliranten Syndromen. Häufig entsteht erheblicher subjektiver Leidensdruck.

Für die Klassifikation, das Verständnis und erst recht die Therapie fehlen zurzeit allgemein akzeptierte Konzepte. Die internationale Literatur ist zu diesem Thema sehr dünn. Dies ist auch nicht verwunderlich, da die historischen Gegebenheiten in wenigen anderen Ländern zu einer ähnlichen Dichte von Belastungen geführt haben. Zwar gibt es zu posttraumatischen Störungen von Holocaust-Überlebenden einzelne hochinteressante Übersichten $[2,3]$, jedoch ist bei der Übertragung der Befunde aus dieser in schrecklicher Weise einzigartigen historischen Situation aus vielerlei Gründen größte $\mathrm{Zu}$ rückhaltung geboten.

Die Symptome sind vielfältig, haben aber oft Ähnlichkeit mit denen einer posttraumatischen Belastungsstörung. Daher wurden oft die Konzepte für dieses Krankheitsbild übertragen. Aufgrund epidemiologischer Studien im deutschsprachigen Raum ist die posttraumatische Belastungsstörung auch bei älteren Menschen eine durchaus häufige Erkrankung [4]. Andererseits sind entscheidende Unterschiede nicht zu übersehen. Bei hochbetagten Menschen wird die Symptomatik vielfach von neuropsychiatrischen Veränderungen im Alter überlagert. Neurodegenerative oder neurovas- 
kuläre Veränderungen, die Verhalten und emotionale Verarbeitung verändern können, sind häufig anzutreffen. Zudem ist das hohe Lebensalter geprägt von neuen sozialen, psychischen und körperlichen Belastungsfaktoren. Andererseits verfügen hochbetagte Menschen vielfach über Resilienzmechanismen, die bei jungen Menschen noch nicht so ausgereift sind [5]. Von daher ist es nicht verwunderlich, dass Grenzen zwischen Krankheitsbildern verschwimmen. Zudem ist es nach der langen Latenz zur Traumatisierung meist schwierig, den Anteil, den diese Traumatisierung an der aktuellen Störung hat, klar zu umreißen.

Vor diesem Hintergrund einer hohen klinischen Relevanz und eines noch unscharf definierten Krankheitsbildes haben Autoren der ersten Beiträge des aktuellen Hefts die schwierige Aufgabe übernommen, den Stand der Forschung und Diskussion zusammenzufassen. H. Glaesmer gibt in ihrem Beitrag eine epidemiologische Übersicht über die Häufigkeit von Traumatisierung und beleuchtet kritisch den Zusammenhang mit psychischer und somatischer Morbidität im Alter. Hucklenbroich et al. beschreiben, gestützt auf eine Literaturübersicht und eigene langjährige klinische Erfahrung, die klinische Präsentation und die diagnostische Abgrenzung des Krankheitsbildes, um dann einen Überblick über Therapieoptionen zu geben. M. Noll-Hussong führt schließlich in die seitherigen Forschungsergebnisse zur Pathogenese dieser Krankheitsbilder ein, wobei er einen besonderen Schwerpunkt auf den Zusammenhang zwischen somatischen Beschwerden und früher Traumatisierung legt.

Neben dem Literaturüberblick finden Sie in der aktuellen Ausgabe eine Originalarbeit von M. Boettche et al., die sich mit einem speziell für ältere Menschen mit Kriegstraumatisierung entwickelten therapeutischen Ansatz beschäftigt.

Wir wünschen bei der Lektüre dieser Artikel interessante neue Einsichten, die Wege zum Verständnis im klinischen Kontext eröffnen, aber auch die wissenschaftliche Diskussion und weitere Forschungsprojekte stimulieren.

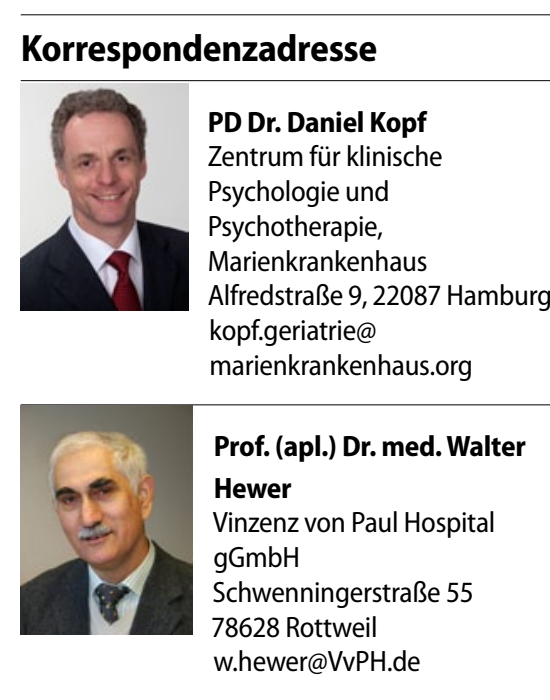

\section{Einhaltung ethischer Richtlinien}

Interessenkonflikt: D. Kopf und W. Hewer geben an, dass kein Interessenkonflikt besteht.

\section{Literatur}

1. Reichling $G$ (1989) Die deutschen Vertriebenen in Zahlen. Teil 2. 40 Jahre Eingliederung in der Bundesrepublik Deutschland. Kulturstiftung der Deutschen Vertriebenen, Bonn, S 34-35

2. Yehuda R, Bierer LM (2008) Transgenerational transmission of cortisol and PTSD risk. Prog Brain Res 167:121-135

3. Golier JA, Harvey PD, Legge J et al (2006) Memory performance in older trauma survivors: implications for the longitudinal course of PTSD. Ann NY Acad Sci 1071:54-66

4. Kuwert P, Knaevelsrud C (2013) Gerontopsychotraumatologie. In: Maercker A (Hrsg.) Posttraumatische Belastungsstörungen, 4. Aufl. Springer, Berlin Heidelberg New York, S 455-468

5. Charles ST, Carstensen LL (2010) Social and emotional aging. Annu Rev Psychol 61:383-409

6. Chaussy U (1982) Jugend. In: Benz W (Hrsg.) Die Bundesrepublik Deutschland. Geschichte in drei Bänden. Bd 2: Gesellschaft. Fischer, Frankfurt, S 35-67 